\title{
LETTER OPEN IFN- $\gamma$ surmounts PD-L1/PD1 inhibition to CAR-T cell therapy by upregulating ICAM-1 on tumor cells
}

Signal Transduction and Targeted Therapy (2021)6:20

\section{Dear Editor,}

Chimeric antigen receptor modified T (CAR-T) cell therapy has shown potent antitumor activity against relapsed and refractory hematological malignancies. However, its efficacy in solid tumors is limited, partly because of the inhibition of PD-L1/PD-1 signaling on CAR-T cells in solid tumors. Further optimizations of CAR-T cells by disrupting PD-1 signaling have improved the anti-tumor efficacy of CAR-T cells. ${ }^{2}$ Here, we report an alternative approach that sensitizes tumor cells by interferon (IFN)- - , but without modifying $\mathrm{T}$ cells; this strategy surmounts PD- 1 inhibition and markedly enhances CAR-T anti-tumor activities in vitro and in vivo.

We constructed two CAR-T cells containing CD28 or 4-1BB costimulatory receptor intracellular domains in tandem with $\mathrm{CD} 3 \zeta$ that target the tumor antigens of HER2 and mesothelin (MSLN) (Supplementary Fig. S1a). Both HER2 and MSLN CAR-T cells exhibited high rates of cytotoxicity to targeted tumor cells, with robust secretion of IFN- $\gamma$ and interleukin (IL)-2 (Supplementary Fig. $\mathrm{S} 1 \mathrm{~b}, \mathrm{c}$ ) and dramatically increased expression of effector activity surface molecule CD107a and intracellular granzyme B (Supplementary Fig. S1d). PD-L1 was significantly upregulated on tumor cells, and coinhibitory molecules including PD-1, TIM-3, and LAG-3 were markedly elevated on CAR-T cells (Supplementary Fig. $\mathrm{S} 2 \mathrm{a}-\mathrm{C}$ ). Moreover, the recursive antigen encountering of HER2 CAR-T cells, mimicking the in vivo antitumor milieu where CAR-T cells serially encounter and kill tumor cells (Supplementary Fig. S3a), showed high cytotoxicity and IFN- $\gamma$ release (Supplementary Fig. S3b), despite high PD-L1 expression on almost all tumor cells (Supplementary Fig. S3c). These results indicated the existence of an intrinsic mechanism of CAR-T cells that surmounts the inhibitory effects of PDL1/PD-1 during tumor cell killing.

IFN- $\gamma$ is an important inducer of PD-L1 expression on tumor cells. ${ }^{3}$ To evaluate the effects of IFN- $\gamma$-induced PD-L1 expression on CAR-T cytotoxicity, tumor cells were primed with IFN- $\gamma$ for $24 \mathrm{~h}$ before exposure to CAR-T cells. IFN- $\gamma$ concentration was optimized as $<10 \mathrm{ng} / \mathrm{ml}$ to ensure induction of PD-L1 expression without inhibiting tumor cell growth (Supplementary Fig. S4a, b). Notably, however, CAR-T cells showed more potent cytolytic activity and IFN- $\gamma$ secretion when exposed to IFN- $\gamma$-primed tumor cells with high expression of PD-L1 (Fig. 1a, b). Conversely, when IFN- $\gamma$ was neutralized by anti-IFN- $\gamma$ antibody, the cytotoxicity of CAR-T cells was significantly attenuated (Fig. 1C), which indicates that IFN- $\gamma$ was critical to CAR-T activities. Notably, in these tests, the medium was replaced with fresh medium to eliminate residual IFN- $\gamma$ $(<10 \mathrm{pg} / \mathrm{ml}$, data not shown), excluding the direct effect of IFN- $\gamma$ on CAR-T cells. We thus speculated that IFN- $\gamma$ functioned to CAR-T activity by acting on tumor cells. To examine this possibility, we first primed tumor cells with low concentrations of IFN- $\gamma$ and measured the cytotoxicity of CAR-T in fresh medium containing sufficient anti-IFN- $\gamma$ antibody to neutralize IFN- $\gamma$ from CAR-T (Fig. 1d). CAR-T cells still exhibited similar killing activity to IFN- $\gamma^{-}$ primed tumor cells when IFN- $\gamma$ was neutralized (Fig. 1e). Second,

\author{
; https://doi.org/10.1038/s41392-020-00357-7
}

we disrupted the IFN- $\gamma R 2$ gene (IFNGR2) in tumor cells using the CRISPR-Cas9 system to block IFN- $\gamma$ signaling (Supplementary Fig. $\mathrm{S} 5 \mathrm{a}-\mathrm{C}$ ), because IFN- $\gamma \mathrm{R} 2$ has been shown to determine the extent of IFN- $\gamma$-induced signaling in given cell populations. ${ }^{4}$ IFN- $\gamma$ R2-null tumor cells grew similarly to control cells (Supplementary Fig. S5d) and did not express PD-L1 any more under IFN- $\gamma$ treatment (Supplementary Fig. S5e). However, these cells were more resistant to the cytolysis of CAR-T cells (Fig. 1f). The enhancement of CAR-T cytotoxicity by IFN- $\gamma$ priming was also significantly diminished as shown by two different target tumor cell lines (Fig. 1g). These results indicated that IFN- $\gamma$ on tumor cells is involved in the intrinsic mechanism of CAR-T to circumvent PD-L1/PD-1.

To directly characterize the function of IFN- $\gamma$ in surmounting PD-L1/PD-1, we tested CAR-T activities on tumor cells that stably overexpress PD-L1. Overexpression of PD-L1 on tumor cells (Supplementary Fig. S6) did not render tumor cells resistant to CAR-T killing (Fig. 1h), and also did not impair the enhancement of CAR-T cytotoxicity to IFN- $\gamma$-primed tumor cells (Fig. 1i). Notably, however, PD-L1 overexpression in IFN- $\gamma$ R2-null tumor cells significantly suppressed both cytolytic activity (Fig. 1j) and secretion of IFN- $\gamma$ and IL-2 (Fig. 1k). These results indicated that IFN- $\gamma$ signaling on tumor cells enhances CAR-T activities by surmounting the inhibitory effects of PD-L1/PD-1. In contrast, the inhibitory function of PD-L1/PD-1 on CAR-T activity requires the absence of IFN- $\gamma$ signaling in tumor cells. This is consistent with the previous study that defects of the interferon signaling pathway are associated with acquired resistance to immune checkpoint blockade therapy. ${ }^{5}$

We next investigated how IFN- $\gamma$ surmounts PD-L1/PD-1 inhibitory effects on CAR-T. IFN- $\gamma$ induces complex molecular events beyond PD-L1 overexpression in tumor cells. We first examined whether IFN- $\gamma$ increased HER2 expression. However, HER2 expression was decreased in IFN- $\gamma$-primed tumor cells (Supplementary Fig. S7), which indicates that HER2 was not involved in IFN- $\gamma$-mediated effects on CAR-T. We found that IFN- $\gamma$ induced intercellular adhesion molecule 1 (ICAM-1, also known as CD54) overexpression on multiple kinds of solid tumor cells (Supplementary Fig. S8a), and LFA-1 (the $\beta 2$ integrin adhesion molecule) was significantly elevated on CAR-T cells after tumor cell exposure (Supplementary Fig. S8b). ICAM-1 is a cell surface glycoprotein on antigen-presenting cells (APCs) and plays a critical role during an effective immune response. The interaction of antigen-laden APCs with CD8 T cells is mediated by the interaction of ICAM-1 to its receptor LFA-1 on T cells. The ICAM-1/LFA-1 interaction promotes efficient adhesion of T cells with APCs and transmits intracellular signals to promote T-cell activation and proliferation. ${ }^{6}$ We then investigated the function of ICAM-1 on CAR-T activity. Knockout of ICAM-1 (Supplementary Fig. S9) in tumor cells with functional IFN- $\gamma$ receptor almost completely abolished the increase of CAR-T cytotoxicity to IFN- $\gamma$ primed tumor cells (Fig. 1I), which indicates the critical role of 
a

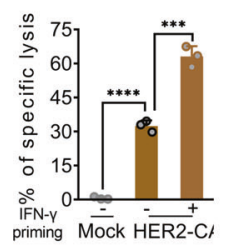

b

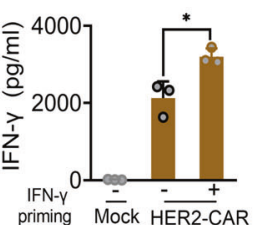

b

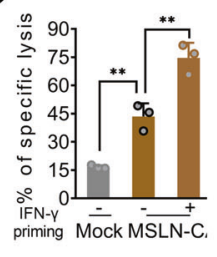

C

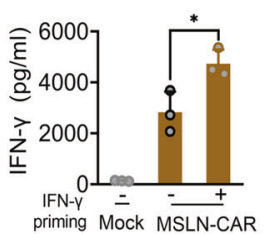

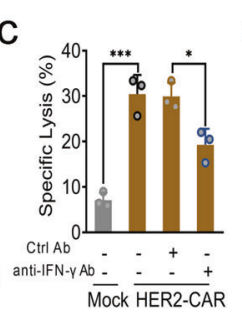

d

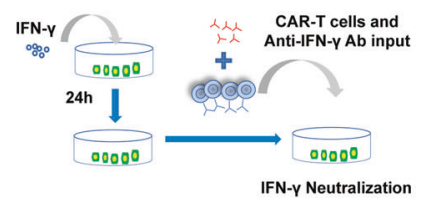

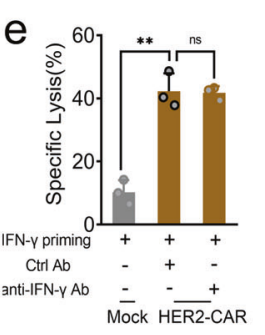

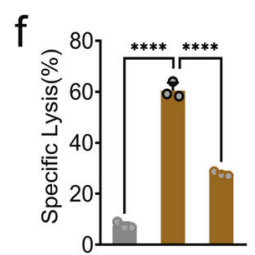

IFN-YR2 WT WT KO Mock HER2-CAR

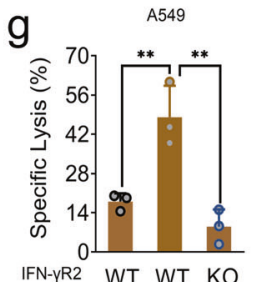

IFN-yR2 WT WT KO
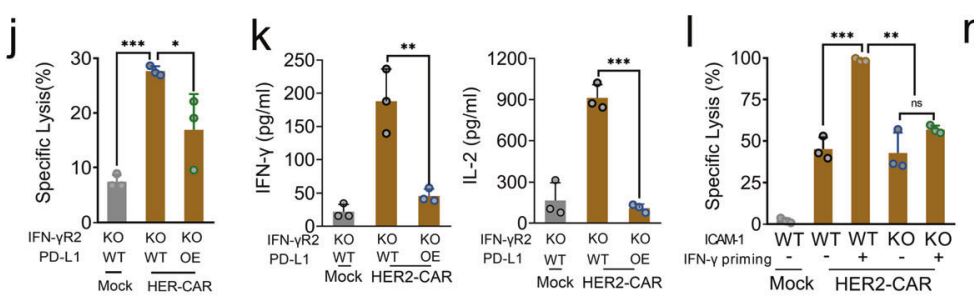

m
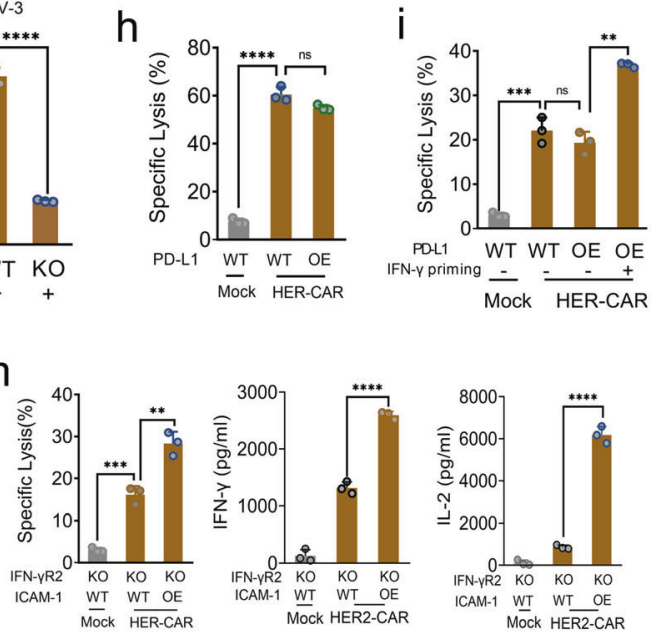

$\mathrm{n}$
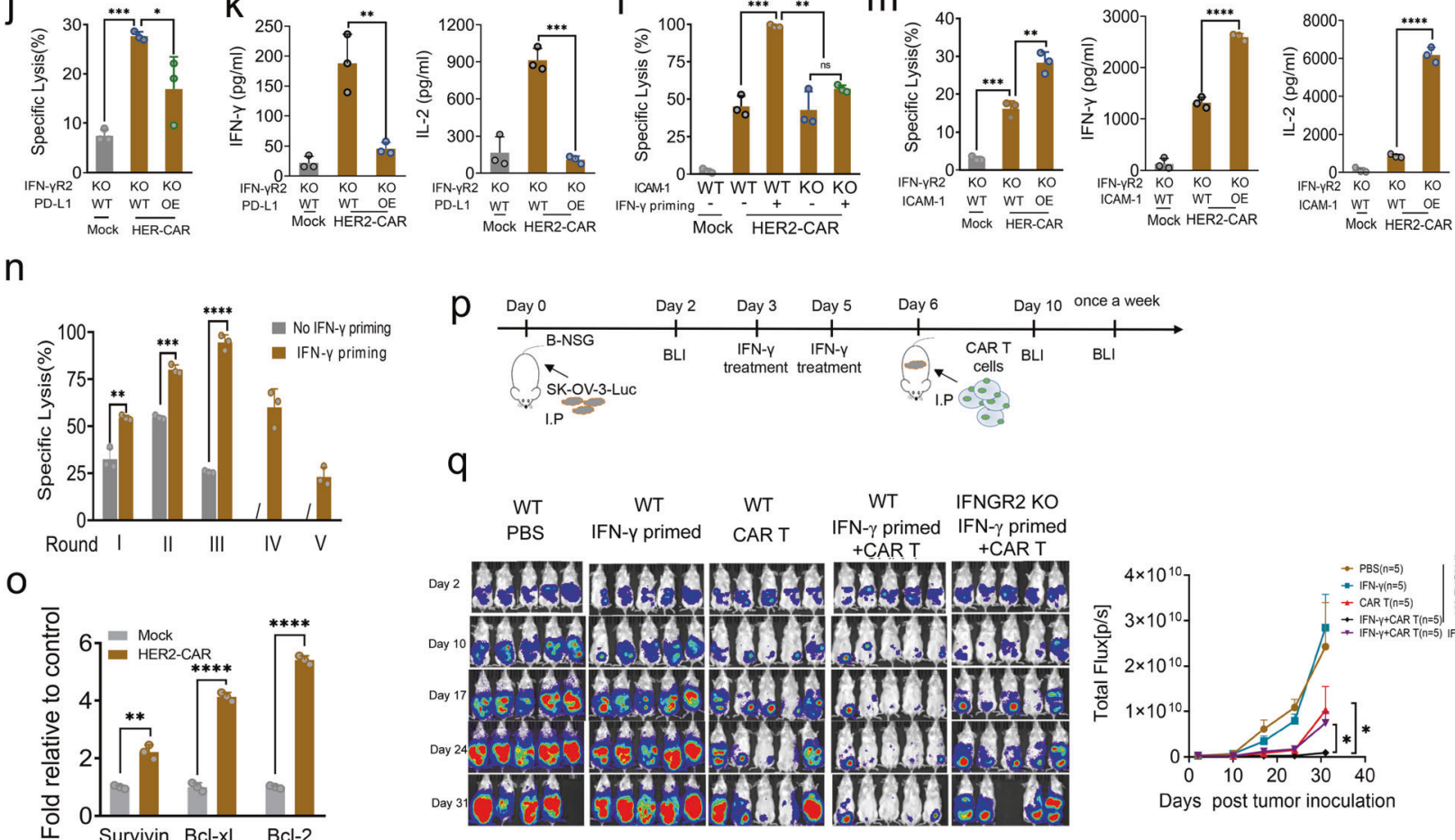

0
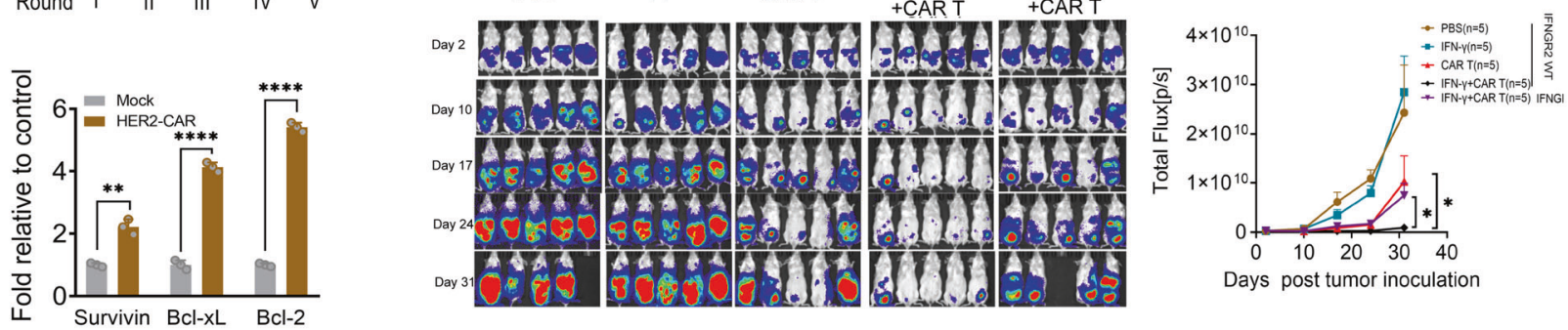

Fig. 1 IFN- $\gamma$ enhances CAR-T anti-tumor activities by sensitizing tumor cells expressing ICAM-1. $\mathbf{a}, \mathbf{b}$ IFN- $\gamma$ priming to tumor cells enhances the specific cytolytic ability and IFN- $\gamma$ secretion of HER2-CAR (a) and MSLN-CAR T (b). c The blockage to IFN- $\gamma$ by using neutralizing antibody decreases the lytic ability of HER2-CAR T cells. d, e Tumor cells were primed by IFN- $\gamma$ for 24 , then were washed using fresh culture media to remove IFN- $\gamma$. Sufficient anti-IFN- $\gamma$ neutralizing antibody was used to block IFN- $\gamma$ release by CAR-T cells (d). The specific lysis was measured by LDH assay (e). f IFN- $\gamma$ R2 knockout in tumor cells significantly decreased CAR-T cytotoxicity. $\mathbf{g}$ IFN- $\gamma$ R2 knockout impaired the enhancement of IFN- $\gamma$-priming to CAR-T cytotoxicities. h PD-L1-overexpression did not decrease CAR-T cytotoxicity to tumor cells. $\mathbf{i}$ IFN- $\gamma$-priming enhanced CAR-T-specific lysis to tumor cells overexpressing PD-L1. j, k PD-L1-overexpression in IFN- $\gamma$ R2-deficient tumor cells inhibited CAR-T cytotoxicity (j) and cytokines release (k). I The knockout of ICAM-1 in tumor cells impaired the enhancement of IFN- $\gamma$-priming to CAR-T cytotoxicity. $\mathbf{m}$ The overexpression of ICAM-1 in IFN- $\gamma$ R2 KO tumor cells enhanced CAR-T cytotoxicity and the secretion of IFN- $\gamma$ and IL-2. $\mathbf{n}$ The enhancement of IFN- $\gamma$ priming to the serial cytotoxic ability of CAR-T cells. o The relatively increased RNA expression of Bcl-2, Bcl-xl, survivin in CAR-T cells that encountered IFN- $\gamma$-primed tumor cells. $\mathbf{p}$ The procedure of combinational therapy of IFN- $\gamma$ and HER2-CAR T cells in peritoneal ovarian cancer models in B-NSG mice. IFN- $\gamma$ was administrated ( $25 \mu \mathrm{g}$ per mouse) prior to CAR-T cells. IP, intraperitoneal injection. q Bioluminescent images and quantitative analysis of peritoneal metastases of human ovarian cancer in selected timepoints during the experiments. Bars represent the mean \pm s.e.m. $p$-values determined by unpaired two-tailed $t$-tests. ${ }^{*} p<0.05,{ }^{* *} p<0.01,{ }^{* *} p<0.001$

ICAM-1 for CAR-T activity. We also ectopically overexpressed ICAM-1 on tumor cells (Supplementary Fig. S10); however, it did not promote the specific cytolysis of CAR-T to tumor cells (Supplementary Fig. S11). We speculated this was because of the elevation of ICAM-1 induced by IFN- $\gamma$ from activated CAR-T cells.
We then overexpressed ICAM-1 in IFN- $\gamma$ R2-knockout cells and found that ICAM-1 overexpression made IFN- $\gamma R 2$-deficient tumor cells more vulnerable to CAR-T killing and more cytokine release (Fig. $1 \mathrm{~m}$ ). Altogether, these results indicated that IFN- $\gamma$-induced ICAM-1 expression underlies the mechanism by which IFN- $\gamma$ 
surmounts PD-L1/PD-1 to sustain or enhance CAR-T anti-tumor activities.

We next tested whether IFN- $\gamma$ priming could help CAR-T achieve better therapeutic effects to solid tumors. In vitro serial killing experiments showed that CAR-T cells maintained cytolytic activity to IFN- $\gamma$-primed tumor cells until round five, compared with round three for control tumor cells (Fig. 1n). Anti-apoptosis genes including bcl-2, bcl-xl, and survivin were significantly upregulated in CAR-T cells that encountered IFN- $\gamma$ primed tumor cells (Fig. 10). Next, we established a peritoneal ovarian cancer model in NSG mice and administrated IFN- $\gamma$ twice before CAR-T cells were intraperitoneally injected (Fig. $1 \mathrm{p})$. Bioluminescence imaging showed that IFN $-\gamma$ was ineffective in inhibiting tumor growth, while CAR-T only delayed tumor growth. However, IFN- $\gamma$ pretreatment combined with CAR-T cells led to elimination of tumors in three of five mice. The remaining two tumors were much smaller than controls (Fig. 1q). However, when IFN- $\gamma$ R2 was deficient, tumors were resistant to the combination therapy. Collectively, these in vitro and in vivo results indicated that sequential therapy using IFN- $\gamma$ and CAR-T would be an effective strategy for solid tumors in the clinic.

In summary, we demonstrated that although IFN- $\gamma$ induces PD$\mathrm{L} 1$ expression in tumor cells, IFN- $\gamma$ is indispensable for CAR-T cell activities by inducing ICAM-1 expression to surmount PD-L1/PD1. The sequential therapy of IFN- $\gamma$ with CAR-T could achieve better therapeutic effects in solid tumors. While current efforts to improve CAR-T therapy to solid tumors have focused on modification of $T$ cells, here we propose that sensitizing tumor cells is also an effective and feasible approach.

\section{DATA AVAILABILITY}

The authors declare that the data supporting the findings of this study are available within the paper and its supplementary information files.

\section{ACKNOWLEDGEMENTS}

This study was supported by National Science and Technology Major Project 2018ZX09733001-004-003, National Natural Science Foundation of China 81772605, and the 1.3.5 project for disciplines of excellence, West China Hospital, Sichuan University (ZYJC18008).

\section{ADDITIONAL INFORMATION}

The online version of this article (https://doi.org/10.1038/s41392-020-00357-7) contains supplementary material, which is available to authorized users.

Competing interests: The authors declare no competing interests.

E Dong ${ }^{1}$, Xiao-zhu Yue ${ }^{1}$, Lin Shui ${ }^{1}$, Bin-rui Liu ${ }^{1}$, Qi-qi Li ${ }^{1}$, Yun Yang ${ }^{1}$, Hui Luo ${ }^{1}$, Wei Wang (D) $^{1}$ and Han-shuo Yang ${ }^{1}$

${ }^{1}$ State Key Laboratory of Biotherapy and Cancer Center, West China Hospital, Sichuan University and Collaborative Innovation Center for Biotherapy, 610041 Chengdu, China

These authors contributed equally: E Dong, Xiao-zhu Yue, Lin Shui Correspondence: Wei Wang (weiwang@scu.edu.cn) or Han-shuo Yang (yhansh@scu.edu.cn)

\section{REFERENCES}

1. Jackson, H. J., Rafiq, S. \& Brentjens, R. J. Driving CAR T-cells forward. Nat. Rev. Clin. Oncol. 13, 370-383 (2016).

2. Michael, A. M. \& Axel, S. Engineering CAR-T Cells for improved function against solid tumors. Front. Immunol. 9, 2493 (2018).

3. Garcia-Diaz, A. et al. Interferon receptor signaling pathways regulating PD-L1 and PD-L2 expression. Cell Rep. 19, 1189-1201 (2017).

4. Rigamonti, L. et al. Surface expression of the IFN-gamma R2 chain is regulated by intracellular trafficking in human T lymphocytes. J. Immunol. 164, 201-207 (2000).

5. Zaretsky, J. M. et al. Mutations associated with acquired resistance to PD-1 blockade in melanoma. N. Engl. J. Med. 375, 819-829 (2016).

6. Scholer, A. et al. Intercellular adhesion molecule-1-dependent stable interactions between T cells and dendritic cells determine CD8+ T cell memory. Immunity $\mathbf{2 8}$, 258-270 (2008).

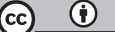

Open Access This article is licensed under a Creative Commons Attribution 4.0 International License, which permits use, sharing, adaptation, distribution and reproduction in any medium or format, as long as you give appropriate credit to the original author(s) and the source, provide a link to the Creative Commons license, and indicate if changes were made. The images or other third party material in this article are included in the article's Creative Commons license, unless indicated otherwise in a credit line to the material. If material is not included in the article's Creative Commons license and your intended use is not permitted by statutory regulation or exceeds the permitted use, you will need to obtain permission directly from the copyright holder. To view a copy of this license, visit http://creativecommons. org/licenses/by/4.0/.

(c) The Author(s) 2021 